05

\title{
Эффект задержки разрушения при разрушении стальных образцов в условиях откола
}

\author{
(ㄱ Н.В. Михайлова, ${ }^{1}$ Г.А. Волков, ${ }^{1,2,9}$ Ю.И. Мещеряков, ${ }^{2}$ Ю.В. Петров, ${ }^{1,2}$ А.А. Уткин ${ }^{1,2}$
}

${ }^{1}$ Санкт-Петербургский государственный университет, 198504 Санкт-Петербург, Петергоф, Россия

${ }^{2}$ Институт проблем машиноведения РАН,

199178 Санкт-Петербург, Россия

ฯ e-mail: volkovgrig@mail.ru; yp@YP1004.spb.edu

(Поступило в Редакцию 15 июня 2016 г.)

Проведены динамические испытания по откольному разрушению двух партий образцов из стали 30ХН4М. Обработка экспериментальных данных проводилась по классической методике, основанной на решении волнового уравнения в упругой постановке. Было выявлено три образца, при испытании которых наблюдался эффект задержки разрушения. При помощи критерия инкубационного времени были показаны условия возникновения задержки разрушения на примере треугольных нагружающих импульсов. Для остальных образцов была построена скоростная кривая прочности. Показано, что предельные напряжения при динамических воздействиях для образцов из разных партий существенно отличаются, несмотря на единый химический состав и одинаковую прочность в статике.

DOI: 10.21883/JTF.2017.04.44312.1931

\section{Введение}

Откольное разрушение является одним из основных методов изучения процессов, протекающих в твердом теле в условиях динамического растяжения [1-5]. Рассмотрение волновых процессов, характерных для откольной задачи, позволяет найти импульс напряжений, приводящий к разрушению [1,2]. Как правило, для оценки параметров откольного импульса используется так называемое акустическое приближение, когда принимаются во внимание только упругие напряжения, действующие в зоне откола.

Разрушение образца может происходить спустя некоторое время после достижения максимума растягивающего напряжения в сечении откола. Это означает, что момент разрушения приходится на ниспадающий или постоянный участок действующего локального напряжения. Данное явление называется задержкой разрушения, а импульсы, соответствующие такому виду разрушения, как правило, являются близкими к пороговым. Под пороговыми понимаются критические импульсы заданной длительности и формы, имеющие минимальную, достаточную для разрушения амплитуду. Задержка разрушения фиксировалась в ряде экспериментов по отколу, например [6,7], однако подробное обсуждение этого явления в литературе практически отсутствует. Нередко в подобной ситуации прочность чисто формально связывают со временем действия растягивающих напряжений в сечении откола [6].

В настоящей работе дается объяснение явлению задержки откольного разрушения с помощью критерия инкубационного времени. Концепция структурно- временного критерия основывается на двух характеристиках материала: квазистатической прочности материала и инкубационном времени разрушения. Указанные величины являются прочностными параметрами материла, которые не зависят от условий эксперимента и могут быть применены для различных видов нагружений. Данный подход показал свою эффективность при определении условий возникновения различных переходных процессов, таких как хрупкое разрушение $[8,9]$, электропробой [10], кавитация в жидкости [11].

\section{Одномерная волновая задача}

Разрушение при отколе происходит следующим образом: ударный импульс создает сжимающую волну в образце, которая продвигается вдоль его оси, достигая свободной поверхности. После отражения от нее волна сжатия меняет знак на противоположный и движется в виде волны растяжения в обратную сторону. Поскольку прочность материалов при растяжении обычно существенно меньше, чем при сжатии, то в некотором сечении образца может произойти разрушение. При пороговых нагрузках оно проявляется в форме зарождения микротрещин. При более интенсивных воздействиях происходит полное отделение от образца части материала в виде так называемой откольной пластины.

Решение одномерной откольной задачи в упругой постановке показывает, что временной профиль волны сжимающих напряжений с точностью до множителя совпадает с $V(t)$ - скоростью движения частиц на свободной поверхности. Таким образом, временная зависимость напряжений в откольном сечении с координатой $x$ 


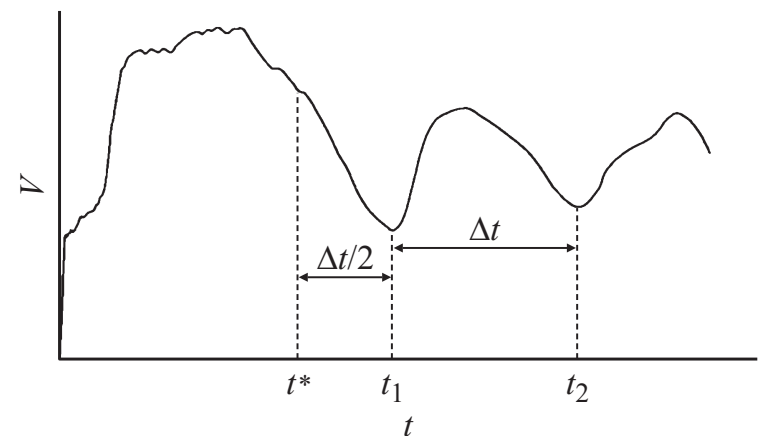

Рис. 1. Зависимость скорости свободной поверхности от времени.

может быть представлена в виде суммы сжимающей и растягивающей волн

$$
\sigma(x, t)=\sigma^{\mathrm{inc}}+\sigma^{\mathrm{ref}}=\frac{1}{2} \rho a V\left(t-\frac{x}{a}\right)-\frac{1}{2} \rho a V\left(t+\frac{x}{a}\right),
$$

где $\rho$ - плотность материала, $a-$ скорость распространения продольной волны напряжения. Следует отметить, что временные профили сжимающей и растягивающей волн полностью совпадают, и при этом растягивающая волна запаздывает на $\Delta t=2 x / a-$ удвоенное время пробега упругой волны по откольному сечению.

При замене $t^{\prime} \rightarrow t-x / a$ временная зависимость в сечении $x^{*}$ может быть записана в виде

$$
\begin{aligned}
\sigma\left(x^{*}, t^{\prime}\right) & =\frac{1}{2} \rho a\left[V\left(t^{\prime}\right)-V\left(t^{\prime}-\frac{2 x^{*}}{a}\right)\right] \\
& =\frac{1}{2} \rho a\left(V_{1}-V_{2}\right)=\frac{1}{2} \rho a \Delta V,
\end{aligned}
$$

т. е. история напряжения в сечении откола до момента разрушения может быть представлена как величина, пропорциональная разности между функцией профиля скорости свободной поверхности и этим же профилем, сдвинутым вправо на величину удвоенного времени пробега волны по толщине откольного слоя [1].

Скорость свободной поверхности образца $V(t)$, как правило, определяется экспериментально методами интерферометрии. Координату откольного сечения $x$ можно вычислить по длительности откольного импульса, совпадающей со временем $\Delta t$ (рис. 1), или просто измерив толщину откольной пластины. В момент времени $t_{1}$ на свободную поверхность приходит сигнал о произошедшем в образце разрушении. Соответственно время самого момента разрушения равно $t^{*}=t_{1}-\Delta t / 2$.

\section{Анализ результатов обработки экспериментальных данных. Эффект задержки разрушения}

Эксперименты по откольному разрушению для настоящей работы проводили в условиях так называемой одноосной деформации, при которой единственной

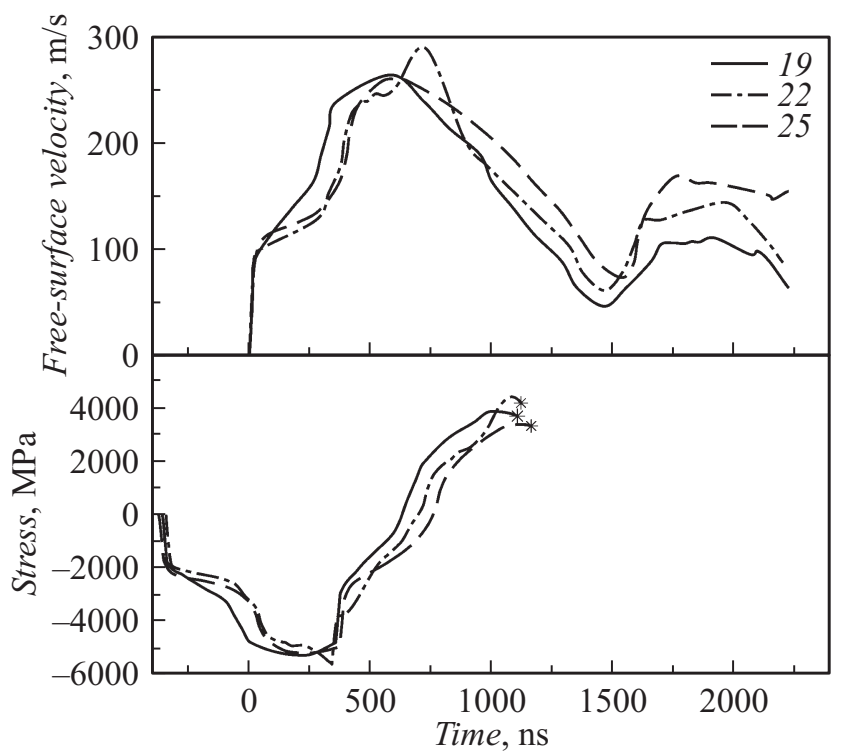

Рис. 2. Временные зависимости для пороговых нагружений скорости свободной поверхности (сверху) и напряжения в сечении откола (снизу).

Таблица 1.

\begin{tabular}{c|c|c|c|c}
\hline № образца & $V_{0}, \mathrm{~m} / \mathrm{s}$ & $\sigma_{\max }, \mathrm{MPa}$ & $\sigma^{*}, \mathrm{MPa}$ & $T_{z}, \mathrm{~ns}$ \\
\hline 19 & 350 & 3886 & 3700 & 100 \\
22 & 365 & 4433 & 4204 & 40 \\
25 & 365 & 3398 & 3340 & 41
\end{tabular}

ненулевой компонентой тензора деформации является компонента, совпадающая с направлением распространения волны. Испытывались две серии образцов из стали 30ХН4М, произведенных при различных условиях термомеханической обработки. Анализ полученных данных проводился, согласно приведенному выше решению волновой задачи (1), в которой профиль напряжений в сечении откола построен со сдвигом на время $\Delta t / 2$.

В случае пороговых нагружений разрушение может происходить с задержкой, через некоторое время после достижения максимальных напряжений в сечении откола (рис. 2). В настоящих экспериментах эффект задержки разрушения наблюдался для образцов 19, 22 и 25 (табл. 1). При скорости ударника $350 \mathrm{~m} / \mathrm{s}$ и толщине образца $1.831 \mathrm{~mm}$ максимальная величина растягивающего напряжения достигает значения $3886 \mathrm{MPa}$. Однако, как следует из экспериментальных данных, образец разрушается при напряжении $3700 \mathrm{MPa}$. При этом время задержки разрушения составляет величину, равную $100 \mathrm{~ns}$.

\section{Критерий инкубационного времени}

Применение критерия инкубационного времени позволяет объяснить многие эффекты, наблюдаемые в динамических испытаниях [8-15]. В частности, явление 
задержки разрушения хорошо согласуется с основной идеей структурно-временного подхода, о том, что возникновению разрыва на данном масштабном уровне обязательно предшествует подготовительный процесс развития дефектов в структуре материла, определяемый некоторыми характерными временами. Поэтому разрушение в сечении откола определятся не мгновенными значениями растягивающих напряжений, а сочетанием амплитудных и временных характеристик локального силового поля. Для материалов без макроскопических дефектов критерий инкубационного времени имеет вид (см., например, [14])

$$
\int_{t-\tau}^{t} \sigma(s, x) d s<\sigma_{c} \tau,
$$

где $\sigma(s, x)$ - история напряжений в сечении $x, \sigma_{c}-$ статическая прочность материала, а $\tau$ - инкубационное (структурное) время разрушения. Таким образом, для того чтобы произошло разрушение в данном сечении, нужно накопить величину импульса не меньше чем $\sigma_{c} \tau$. Параметры $\sigma_{c}$ и $\tau$ являются константами материала и не зависят от геометрии исследуемого образца, а также от формы и продолжительности воздействия. Таким образом, можно говорить о том, что статическая прочность $\sigma_{c}$ и инкубационное время $\tau$ образуют систему определяющих параметров процесса разрушения.

Экспериментально измеренные временные зависимости скорости свободной поверхности позволяют полностью восстановить профиль напряжений в откольном сечении вплоть до момента разрушения $t^{*}$. Таким образом, величина инкубационного времени $\tau$ в каждом конкретном испытании может быть определена путем непосредственной подстановки вычисленных напряжений в критерий (2). Результаты вычислений приведены в табл. 2.

Обработка данных для запороговых испытаний показала, что напряжения на стадии растяжения образца можно приближенно описывать линейной зависимостью

$$
\sigma(t)=\dot{\sigma} t H(t)
$$

После подстановки нагрузки линейного вида (3) в критерий (2) и последующего интегрирования можно получить простое выражение для вычисления инкубационного времени

$$
\tau=2\left(t^{*}-\frac{\sigma_{c}}{\dot{\sigma}}\right) .
$$

Вычисление инкубационного времени по формуле (4) и расчеты, проведенные ранее, дали сопоставимые результаты, тем самым подтверждая предположение о линейности роста растягивающих напряжений в проведенных испытаниях. Поэтому для определения некоторого среднего интегрального значения инкубационного времени разрушения строились прочностные кривые $\sigma(\dot{\sigma})$,

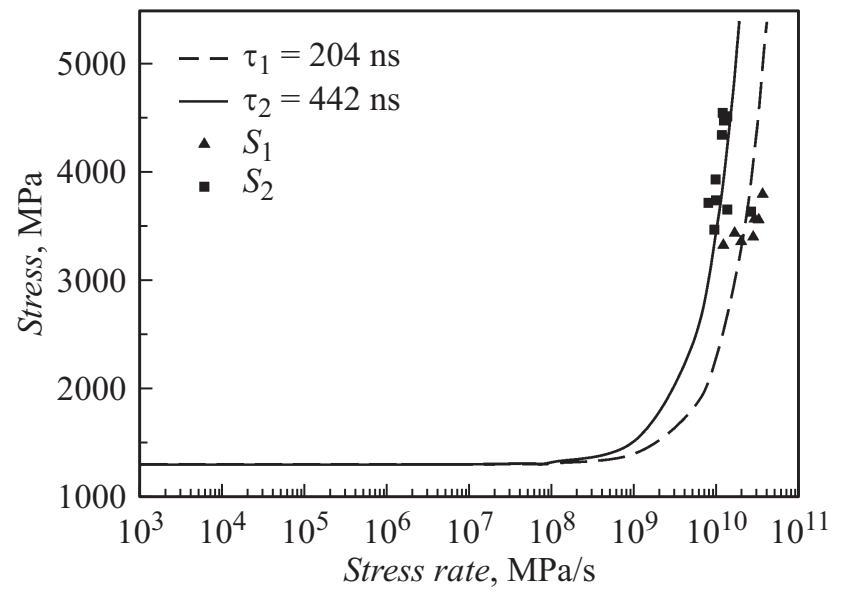

Рис. 3. Скоростная зависимость предельных напряжений, полученных для $\tau_{1}=204 \mathrm{~ns}$ и $\tau_{2}=442 \mathrm{~ns}$, и зависимость $\sigma(\dot{\sigma})$ для экспериментальных точек. Статическая прочность $\sigma_{c}=1300 \mathrm{MPa}$.

соответствующие разным $\tau$. Далее методом наименьших квадратов определялась кривая, наиболее совпадающая с экспериментальными точками, а соответствующее этой кривой значение $\tau$ было принято за инкубационное время разрушения для исследуемого материала. В результате для каждой из партий стали получено два значения инкубационного времени: $\tau_{1}=204 \mathrm{~ns}$ и $\tau_{2}=442 \mathrm{~ns}$ (рис. 3). Оказалось, что образцы из одной партии имеют большую динамическую прочность при одинаковой прочности в статике $\sigma_{c}=1300 \mathrm{MPa}$.

\section{Явление переотражения волн. Реализация эффекта задержки разрушения}

Использование структурно-временного подхода позволяет найти условия, при которых разрушение будет происходить с задержкой. Согласно критерию инкубационного времени, увеличение амплитуды или продолжительности порогового импульса приводит к разрушению. Это обстоятельство коренным образом отличает процесс динамического разрушения от медленного, квазистатического, при котором к материалу нельзя приложить нагрузку, которая превышает его статическую прочность $\sigma_{c}$. Поэтому представляется правильным при ударном нагружении находить пороговые нагрузки в виде импульсов определенной формы с фиксированными значениями амплитуды и длительности. Это влечет проведение целой серии испытаний, в которых при заданных длительности и форме нагрузки определяется минимально необходимая для разрушения амплитуда. Подобные эксперименты проводились в [15], где наблюдался задержанный старт макроскопических трещин на ниспадающей ветви локального силового поля, т.е. момент разрушения наступал спустя некоторое время 
Таблица 2.

\begin{tabular}{|c|c|c|c|c|c|}
\hline \multicolumn{3}{|c|}{$\begin{array}{c}\text { Первая серия образцов } \\
\left(\tau_{1}=204 \mathrm{~ns}\right)\end{array}$} & \multicolumn{3}{|c|}{$\begin{array}{c}\text { Вторая серия образцов } \\
\left(\tau_{2}=442 \mathrm{~ns}\right)\end{array}$} \\
\hline$\tau, \mathrm{ns}$ & $\dot{\sigma} \cdot 10^{10}, \mathrm{MPa} / \mathrm{s}$ & $\sigma^{*}, \mathrm{MPa}$ & $\tau, \mathrm{ns}$ & $\dot{\sigma} \cdot 10^{10}, \mathrm{MPa} / \mathrm{s}$ & $\sigma^{*}, \mathrm{MPa}$ \\
\hline 232 & 1.95 & 3355 & 598 & 0.778 & 3710 \\
\hline 301 & 1.62 & 3432 & 195 & 2.580 & 3630 \\
\hline 401 & 1.19 & 3322 & 566 & 1.200 & 4470 \\
\hline 188 & 2.83 & 3563 & 477 & 1.320 & 4507 \\
\hline 174 & 3.18 & 3555 & 561 & 0.958 & 3926 \\
\hline 169 & 3.55 & 3791 & 508 & 0.963 & 3734 \\
\hline \multirow[t]{4}{*}{169} & 2.73 & 3397 & 567 & 1.140 & 4336 \\
\hline & & & 597 & 1.160 & 4541 \\
\hline & & & 576 & 0.924 & 3463 \\
\hline & & & 406 & 1.320 & 3650 \\
\hline
\end{tabular}

после прохождения коэффициентом интенсивности максимального значения.

Наиболее наглядно условия возникновения эффекта задержки разрушения можно продемонстрировать на примере нагружающих импульсов треугольной формы. Пусть $P$ - амплитуда импульса, $T=T_{1}+T_{2}-$ его продолжительность, $T_{1}$ и $T_{2}$ - длительности участков роста и спада нагрузки соответственно. Если импульс пороговый, то разрушение всегда будет происходить на участке спада нагрузки, т. е. будет наблюдаться задержка разрушения. Из критерия (2) следует, что амплитуда такого импульса

$$
P_{\text {th }}= \begin{cases}\frac{2 \sigma_{c} \tau}{T}, & T<\tau, \\ \frac{2 \sigma_{c} T}{2 T-\tau}, & T>\tau .\end{cases}
$$

Для времени задержки разрушения $T_{d}$ будет справедливо следующее выражение

$$
T_{d}= \begin{cases}T_{2}, & T<\tau, \\ \frac{T_{2} \cdot \tau}{T}, & T>\tau .\end{cases}
$$

Увеличение амплитуды импульса с неизменными продолжительностями роста и спада $T_{1}$ и $T_{2}$ сделает его запороговым. Отношение амплитуд запорогового импульса к минимальному разрушающему характеризует величину перегрузки. Для запороговых импульсов, длительность которых не превосходит величины инкубационного времени $\tau$, увеличение амплитуды приведет к уменьшению времени задержки. При этом, если участок нагружения короче спада, задержка разрушения будет происходить при любом перегрузе. В противном случае время задержки будет уменьшаться до нуля, и амплитуда, соответствующая этому моменту, будет определяться следующим выражением: $P=2 \sigma_{c} \tau /\left(T_{1}-T_{2}\right)$. Дальнейшее увеличение амплитуды приводит к тому, что условие разрушения наступает в двух сечениях одновременно. При этом одно из этих сечений находится на участке роста нагрузки, а в другом напряжение в момент разрушения уменышается. Величины напряжений в обоих сечениях совпадают.
Для импульсов с длительностью, превосходящей величину инкубационного времени $\tau$, а также у которых участок нагружения короче спада $T_{1}<T_{2}$, возможны два случая. Первый вариант - как при воздействии коротких импульсов - разрушение происходит на участке спада нагрузки. Во втором случае моменту разрушения предшествует участок, на котором напряжения не изменяются. Такая ситуация наблюдалась в экспериментах [1]. Продолжительность участка постоянства напряжений можно также трактовать как задержку разрушения, поскольку напряжения не меняются и говорить о скоростной зависимости прочности нельзя. Наконец, если участок нагружения длиннее спада $T_{1}>T_{2}$ и перегруз небольшой, то разрушение происходит с задержкой, т. е. на участке спада нагрузки. При увеличении перегруза разрушение возникает во время возрастания уровня напряжений и задержки разрушения не наблюдается.

На рис. 4 представлены нормированные напряжения в сечении откола в зависимости от нормированного времени в различных ситуациях: $a-T<\tau, T_{1}<T_{2}$; $b-T<\tau, T_{1}>T_{2} ; c-T>\tau, T_{1}<T_{2} ; d-T>\tau$, $T_{1}>T_{2}$. На рис. $4, b$ два сечения разрушения - штриховой линией с большей координатой, а на рис. 4, $c$ штрихпунктирной - падающий, штриховой - отраженный и сплошной линией - суммарное напряжение. Кружком обозначается момент разрушения.

Таким образом, задержки разрушения не будет только при нагружении импульсами, продолжительность участка роста нагрузки которых длиннее спада и при больших перегрузках. При разрушении воздействиями произвольной формы общая картина, наблюдаемая при воздействии рассмотренными выше импульсами „идеальной формы, сохранится. Для импульса любой формы есть минимальная пороговая амплитуда, при которой будет наблюдаться задержка разрушения. Увеличение амплитуды вызовет уменьшение времени задержки. Следовательно, момент разрушения относительно начала действия приложенного импульса уменьшится. Если приложенный импульс немонотонный, то момент разрушения 

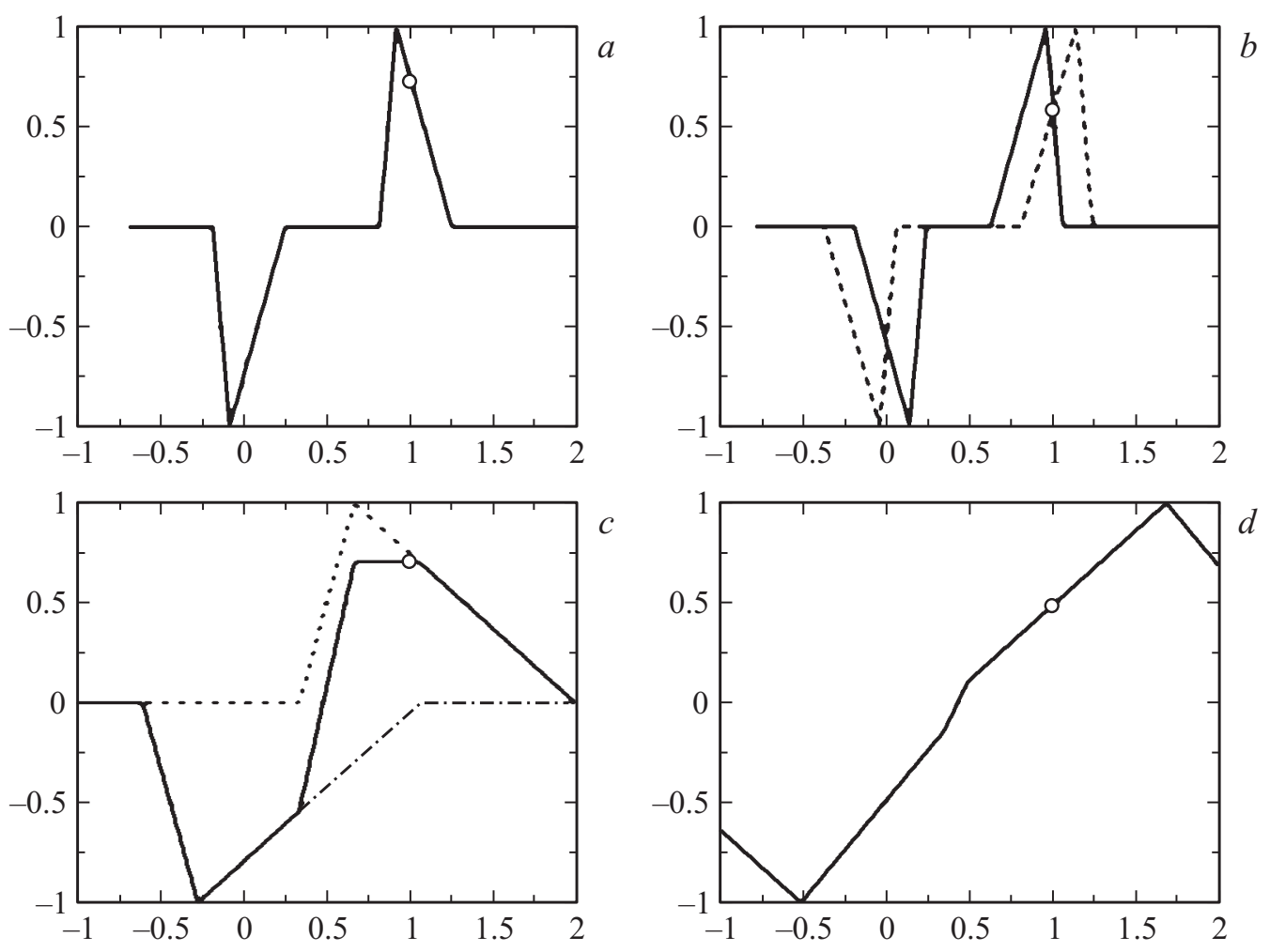

Pис. 4. Напряжения в сечении откола при различных соотношениях длительностей участков нагружения и спада нагрузки.

при увеличении амплитуды может начать увеличиваться. Возможно также возникновение условия разрушения в нескольких сечениях одновременно.

Анализ полученных результатов показывает, что условие осуществления эффекта задержки разрушения зависит не только от амплитуды ударного импульса, но и от его продолжительности. В тонких образцах, нагруженными длительными воздействиями, может возникнуть явление переотражения волны. Передний фронт растягивающей волны, отражаясь от нагружаемой поверхности, возвращается в сечение откола, воздействуя на образец уже сжимающими напряжениями. Затем эта волна, дойдя до свободной поверхности, также будет отражаться. Таким образом, напряжение в точке с координатой $x$ будет иметь вид

$$
\begin{aligned}
\sigma(x, t)= & \frac{1}{2} \rho a\left[-V\left(t+\frac{x}{a}\right)+V\left(t-\frac{x}{a}\right)\right. \\
& \left.-V\left(t+\frac{x}{a}-\frac{2 l}{a}\right)+V\left(t-\frac{x}{a}-\frac{2 l}{a}\right)-\ldots\right] .
\end{aligned}
$$

Здесь $l-$ длина образца, $T_{0}=2 l / a-$ время, через которое волна вернется в исходное место в образце. Когда аргумент очередного слагаемого в сумме станет меньше нуля, ряд можно оборвать, так как нагрузка прикладывается в момент времени $t=0$. В результате сложения упругих волн напряжений разных знаков уменьшается интенсивность растягивающего воздействия на образец. Таким образом, суммарный растягивающий импульс не приводит к разрушению материала. Дальнейшее увеличение скорости ударника без изменения его толщины приведет к запороговым нагружениям, при которых возникновение явления задержки разрушения невозможно. Поэтому для получения данного эффекта в экспериментах необходимо точно контролировать соотношение длительности воздействия и толщину образца так, чтобы избежать переотражения растягивающей волны.

\section{Заключение}

В результате анализа экспериментальных данных по откольному разрушению удалось выявить несколько испытаний, в которых наблюдается эффект задержки разрушения. Данный эффект можно объяснить при помощи структурно-временного критерия, который также позволяет рассчитать пороговые напряжения для различных скоростей нагружения. С помощью критерия инкубационного времени найдены прочностные параметры исследуемого материала. Показано, что способ обработки стали может существенно повлиять на ее динамическую прочность. Проведенное исследование позволяет сделать вывод, что для наблюдения эффекта задержки разрушения нужно очень четко контролировать все условия эксперимента и соблюдать определенные соотношения между значениями контрольных параметров: длительностью и амплитудой воздействия, а также толщиной образца. 
Опираясь на полученные результаты, можно сделать следующий вывод. Для изучения прочностных свойств материала предпочтительнее воздействовать на него не только высокоскоростными „перегруженными“ импульсами с высокой плотностью подводимой энергии, но также и пороговыми воздействиями. В таком случае могут наблюдаться временные эффекты, свойственные только для динамического режима разрушения. Если проводить нагружение высокоинтенсивными запороговыми воздействиями, то тогда материал образца вследствие большой скорости роста напряжений не будет успевать проявлять некоторые свойственные ему структурно-временные эффекты разрушения.

Работа выполнена при поддержке грантов РФФИ 17-01-00618, 16-51-53077, 16-01-00638, а также гранта Президента Российской Федерации МК-7596.2015.1.

\section{Список литературы}

[1] Златин Н.А., Пугачев Г.С., Мочалов С.М., Брагов А.М. // ФТТ. 1975. Т. 17. Вып. 9. С. 2599-2602.

[2] Broberg K.B. Cracs and Fracture. London: Academic Press, 1999. $754 \mathrm{p}$.

[3] Батани Д., Вовченко В.И., Кильпио А.В., Красюк И.К., Ломоносов И.В., Пашинин П.П., Семенов А.Ю., Фортов В.Е., Шашков Е.В. // Докл. РАН. 2003. Т. 389. Вып. 3. C. $328-331$

[4] Liqiang Lin, Xiaowei Zeng // Eng. Fract. Mech. 2015. Vol. 142. P. 50-63.

[5] Dalton D.A. et. al. // J. Appl. Phys. 2008. Vol. 104. P. 013526.

[6] Златин Н.А., Пугачев Г.С. и др. // ФТТ. 1974. Т. 16. Вып. 6. C. $1752-1755$.

[7] Златин Н.А., Песчанская Н.Н., Пугачев Г.С. // ЖТФ. 1986. Т. 56. Вып. 2. С. 403-406.

[8] Petrov Y.V. In: Rock Dynamics and Applications - State of the Art / Ed. by J. Zhao and J. Li. London: Taylor \& Francis Group, 2013. P. 101.

[9] Bratov V.A., Gruzdkov A.A., Krivosheev S.I., Petrov Y.V. // Doklady Physics. 2004. Vol. 49. N 5. P. 338-341.

[10] Петров Ю.В. // ДАН. 2014. Т. 454. Вып. 3. С. 289-292.

[11] Груздков А.А., Волков Г.А., Петров Ю.В. // Акустический журн. 2007. Т. 53. Вып. 2. С. 149-152.

[12] Petrov Y.V., Utkin A.A. // Sov. Mater. Sci. 1989. Vol. 25. N 2. P. 153-156.

[13] Морозов Н.Ф., Петров Ю.В., Уткин А.А. // ДАН СССР. 1990. Т. 313. Вып. 2. С. 276-279.

[14] Петров Ю.В., Смирнов И.В., Уткин А.А. // МТТ. 2010. T. 45. Вып. 3. С. 200-210.

[15] Berezkin A.N., Krivosheev S.I., Petrov Y.V., Utkin A.A. // Doklady Physics. 2000. Vol. 45. N 11. P. 617-619. 